\title{
Can the Cancer-Related Fatigue case-definition criteria be applied to chronic medical illness? A comparison between breast cancer and systemic sclerosis
}

Linda Kwakkenbos ${ }^{1,2}$; Ollie Minton ${ }^{3}$; Patrick Charles Stone ${ }^{4}$; Susanna Alexander ${ }^{5}$; Murray Baron $^{2,6}$; Marie Hudson²,6; Brett D. Thombs ${ }^{1,2,6-10}$ and the Canadian Scleroderma Research Group $^{11}$

\begin{abstract}
Objective: Fatigue is a crucial determinant of quality of life across rheumatic diseases, but the lack of agreed-upon standards for identifying clinically significant fatigue hinders research and clinical management. Case definition criteria for Cancer-Related Fatigue were proposed for inclusion in ICD. The objective was to evaluate whether the Cancer-Related Fatigue case definition performs equivalently in women with breast cancer and systemic sclerosis (SSc) and could be used to identify patients with Chronic-Illness Related Fatigue.

Methods: The Cancer-Related Fatigue interview (case definition criteria met if $\geq 5$ of 9 fatiguerelated symptoms present with functional impairment) was completed by 291 women with SSc and 278 women successfully treated for breast cancer. Differential item functioning was assessed with the Multiple-Indicator Multiple-Cause model.
\end{abstract}

Results: Items 3 (concentration) and 10 (short-term memory) were endorsed significantly less often by women with SSc compared to cancer, controlling for responses on other items. Omitting these 2 items from the case definition and requiring 4 out of the 7 remaining symptoms resulted in a similar overall prevalence of Cancer-Related Fatigue in the cancer sample compared to original criteria (37.4\% versus $37.8 \%$, respectively) with $97.5 \%$ of patients diagnosed identically 
with both definitions. Prevalence of Chronic Illness-Related Fatigue was 36.1\% in SSc using 4 of 7 symptoms.

Conclusions: The Cancer-Related Fatigue criteria can be used equivalently to identify patients with Chronic Illness-Related Fatigue when two cognitive fatigue symptoms are omitted. Harmonized definitions and measurement of clinically significant fatigue will advance research and clinical management of fatigue in rheumatic diseases and other conditions.

Key indexing terms: Fatigue; scleroderma, systemic; patient outcome assessment; neoplasms; psychometrics;

${ }^{1}$ Department of Psychiatry, McGill University, Montréal, Québec, Canada; ${ }^{2}$ Lady Davis Institute for Medical Research, Jewish General Hospital, Montréal, Québec, Canada; ${ }^{3}$ Division of Population Health Sciences and Education, St Georges University of London, London, United Kingdom; ${ }^{4}$ Marie Curie Palliative Care Research Department, Division of Psychiatry, University College London, London, United Kingdom; ${ }^{5}$ Norfolk and Norwich University Hospitals NHS Foundation Trust, Norwich, United Kingdom; Departments of ${ }^{6}$ Medicine, ${ }^{7}$ Epidemiology, Biostatistics, and Occupational Health, ${ }^{8}$ Educational and Counselling Psychology, ${ }^{9}$ Psychology, and ${ }^{10}$ School of Nursing, McGill University, Montréal, Québec, Canada; ${ }^{11}$ CSRG Recruiting Rheumatologists who contributed to this study include: M. Baron, Montreal, Quebec; M. Hudson, Montreal, Quebec; N. Khalidi, Hamilton, Ontario; E. Kaminska, Hamilton, Ontario; J. Pope, London, Ontario; J. Markland, Saskatoon, Saskatchewan; N. Jones, Edmonton, Alberta; P. Docherty, Moncton, New Brunswick; J-P. Mathieu, Montreal, Quebec. 
Conflict of interest statement: There are no conflicts of interest to disclose.

Funding statement: This work was supported by Cancer Research UK (Grant \#C11075/A7143 and C31193/ A10090), an American College of Rheumatology Research and Education Foundation Health Professional Investigator Award, and grants from the Fonds de la Recherche en Santé du Québec (dossier \#14409) and the Canadian Institutes of Health Research (CIHR; grant \#TR3-267681). The Canadian Scleroderma Research Group (CSRG) has been funded by the CIHR (grant \#FRN 83518), the Scleroderma Society of Canada and its provincial chapters, Scleroderma Society of Ontario, Sclérodermie Québec, Cure Scleroderma Foundation, INOVA Diagnostics Inc. (San Diego, CA), Dr. Fooke Laboratorien GmbH (Neuss, Germany), Euroimmun (Lubeck, Germany), Mikrogen GmbH (Neuried, Germany), Fonds de la recherche en santé du Québec (FRSQ), the Canadian Arthritis Network, and the Lady Davis Institute of Medical Research of the Jewish General Hospital, Montreal, Quebec. The CSRG has also received eductional grants from Pfizer and Actelion pharmaceuticals. Dr. Kwakkenbos was supported by a Fonds de Recherche du Québec - Santé (FRQS) postdoctoral fellowship. Dr Hudson was supported by a Fonds de Recherche du Québec - Santé (FRQS) Clinical Research Scholar award. Dr. Thombs was supported by an Investigator Salary Award from the Arthritis Society

Linda Kwakkenbos, $\mathrm{PhD}^{1,2}$; Ollie Minton, $\mathrm{PhD}^{3}$; Patrick Charles Stone, $\mathrm{MD}^{4}$; Susanna Alexander, $\mathrm{PhD}^{5}$; Murray Baron, $\mathrm{MD}^{2,6}$; Marie Hudson, $\mathrm{MD}^{2,6}$; Brett D. Thombs, $\mathrm{PhD}^{1,2,6-10}$ and the Canadian Scleroderma Research Group ${ }^{11}$ 
Address for Reprint Requests and Correspondence: Linda Kwakkenbos, PhD; Jewish General Hospital; 4333 Cote Ste Catherine Road; Montreal, Quebec H3T 1E4; Tel: (514) 340-8222 ext. 8578; Email: kwakkenbosL@gmail.com

Suggested running footline: Fatigue case-definition 


\section{INTRODUCTION}

Persistent fatigue from chronic medical disease involves exhaustion disproportionate to exertion, which is not relieved by rest and can have a major impact on health-related quality of life (HRQL) (1,2). Clinicians, however, are often unsure how to address fatigue $(3,4,5)$. Almost $90 \%$ of rheumatologists never assess fatigue (6), and most cancer patients do not discuss fatigue with their physicians $(7,8)$.

Most studies of fatigue in medical illness use single items or scores above a cut-off threshold on continuous scales to define fatigue. These methods, however, are not benchmarked to any case-definition standard and do not necessarily identify clinically significant fatigue levels that warrant investigation and treatment $(9,10)$. Researchers in cancer have developed CancerRelated Fatigue case-definition criteria that have been proposed for inclusion in the International Classification of Diseases (11). Cancer-Related Fatigue caseness is based on four criteria: (1) the presence of $\geq 5$ of 9 fatigue symptoms with 'significant fatigue, lack of energy, or an increased need to rest' on (nearly) every day in a 2-week period in the last month; (2) impact on daily activities; (3) evidence that symptoms are a consequence of cancer or cancer therapy; and (4) not primarily a consequence of comorbid psychiatric disorders (11).

Establishing a common fatigue case-definition across diseases would enhance comparability of research results and could help improve clinical management. The CancerRelated Fatigue case-definition, however, has not been tested in any other patient groups. To apply the criteria more broadly to define Chronic Illness-Related Fatigue, criteria items must be measurement equivalent across disease groups, meaning that patients across groups with similar levels of fatigue will respond similarly to items (12). Differential item functioning (DIF), on the other hand, is said to occur when patients from different disease groups with similar levels of 
fatigue score differently on items assessing fatigue. DIF between disease groups may occur because of underlying differences in item relevance or in the way specific items are perceived or interpreted (13). DIF analyses can help inform whether the Cancer-Related Fatigue casedefinition operates consistently across diseases or whether there may be cross-disease measurement differences that reflect elements of fatigue specific to a certain illness, but less relevant to others.

Patients with systemic sclerosis (SSc, or scleroderma) provide an ideal population to test the viability of the Cancer-Related Fatigue case-definition paradigm as a general fatigue casedefinition for potential use in rheumatic diseases. SSc is a chronic, multi-system connective tissue disorder characterized by thickening and fibrosis of the skin, involvement of internal organs, substantially reduced HRQL, and significant morbidity and mortality $(14,15)$. Fatigue in SSc is common, influences daily function more than any other symptom, and is independently associated with reduced capacity to carry out daily activities, work disability and impaired physical function (16-21).

The objective of this study was to assess the measurement equivalence of the CancerRelated Fatigue case-definition criteria between SSc and breast cancer patients and examine whether this definition can be used to identify patients with SSc with clinically significant fatigue and, potentially more broadly, as a case definition for Chronic Illness-Related Fatigue.

\section{MATERIALS AND METHODS}

\section{Patients and Procedures}

SSc sample. Data for the SSc sample were collected as part of a Canadian Scleroderma Research Group (CSRG) Registry sub-study. Patients with a SSc diagnosis confirmed by a CSRG 
rheumatologist, $\geq 18$ years of age and fluent in English or French were recruited from 7 of 15 CSRG centers. Over $98 \%$ of patients in the Registry meet the 2013 ACR/EULAR SSc classification criteria (22,23). Registry patients annually undergo physical evaluations and complete self-report questionnaires. Participants were recruited during their annual Registry visit to participate in a telephone interview related to fatigue. The present study included data from the first of two interviews conducted between April 2009 and May 2012. We included women, but not men, since we compared data to women with breast cancer. The study was approved by the McGill University Institutional Review Board, and patients provided informed written consent.

Breast cancer sample. Data were collected in two separate studies conducted in a nurseled follow up clinic at St George's Hospital, London, UK. Disease-free women $\geq 18$ years of age diagnosed histologically with breast cancer (stage I-III) were included (24). All women were clinically and radiologically disease-free and between 3 months and 2 years post-completion of primary breast cancer treatment (of any modality). Cancer-Related Fatigue interviews were conducted in person between January 2006 and January 2008, and between January 2009 and June 2011. For both studies, local ethics approval was obtained from Wandsworth regional ethics committee, and all patients provided informed written consent.

\section{Measures}

Demographics and disease characteristics. In SSc patients, time since SSc diagnosis, extent of cutaneous involvement, and current use of immunosuppressive medications (methotrexate, cyclophosphamide, azatrophine, and mycophenylate) were recorded by a CSRG rheumatologist. Limited SSc was defined as skin involvement distal to the elbows and knees only, whereas diffuse SSc was defined as skin involvement proximal to the elbows and knees, and/or the trunk (25). Patients with sine SSc were grouped with limited SSc patients in analyses. 
Cancer comorbidity was self-reported by patients. In the cancer sample, time post-treatment and disease-related data, including histological stage and treatment history, were collected via medical records.

Fatigue interview. The Cancer-Related Fatigue interview determines whether patients have experienced 2 weeks of significant fatigue in the preceding month (Criterion A1) and the presence of $\geq 5$ of 9 fatigue-related symptoms (Criteria A2-A10). In addition, single items assess whether fatigue has significantly affected work or self care (Criterion B), are a consequence of cancer or cancer therapy (Criterion C), and are primarily a consequence of a co-morbid psychiatric disorder, primarily depression (Criterion D) (10). In the SSc sample, Criterion C was not explicitly assessed related to SSc or its treatment. In cancer samples, when Criterion C has been explicitly assessed (24), no patients have been excluded based on non-cancer sources of fatigue, and it seemed unlikely that SSc patients would be excluded on this basis. Although the case definition permit patients with co-existing psychiatric disorders to be classified as cases provided the psychiatric condition is not the primary cause of the fatigue, we excluded all such patients from both samples, as the inclusion of women with co-morbid psychiatric disorders would have made it difficult to interpret the significance of any differences that were identified between the two groups.

Depression interview. In the SSc sample, the Depression Module of the Composite International Diagnostic Interview (CIDI) (26) was administered by trained interviewers to assess whether patients met criteria for current (30-day) Major Depressive Disorder (MDD) based on Diagnostic and Statistical Manual-IV (DSM-IV) criteria (27).

In the one of the cancer samples (28), eligible patients were first screened for psychiatric pathology clinically, and the remaining eligible patients completed the Structured Clinical 
Interview for the DSM-IV (SCID) (29). In the other cancer sample (30), all eligible patients completed the SCID.

\section{Statistical Analyses}

Demographic characteristics were compared between samples using the chi-square statistic for categorical variables and t-tests for continuous variables. The factor structure of Cancer-Related Fatigue criteria items was assessed for each sample separately using confirmatory factor analysis (CFA) with Mplus (31). For DIF assessment, the simplest structure with reasonable fit is used. A previous study demonstrated that Cancer-Related Fatigue can be considered unidimensional for measurement applications, such as DIF analysis (32). Thus, a single-factor CFA model was constructed. Item responses for the case-definition were binary and thus modeled using the weighted least squares estimator with a diagonal weight matrix, robust standard errors, and a mean- and variance-adjusted chi-square statistic with delta parameterization, using full information maximum likelihood for missing data (31). The chisquare test, the Tucker-Lewis Index (TLI) (33), the Comparative Fit Index (CFI) (34) and the Root Mean Square Error of Approximation (RMSEA) (35) were used to assess model fit. Good fitting models are indicated by a TLI and CFI $\geq 0.95$ and RMSEA $\leq 0.06$ (36). A CFI of .90 or above and a RMSEA of .08 or less (37) are also considered acceptable model fit. The chi-square test is highly sensitive to sample size and can lead to the rejection of well-fitting models (38). Therefore, the TLI, CFI and RMSEA were emphasized. Modification indices were used to identify pairs of items for which model fit would improve if errors were freed to covary. Once the factor structure was established for each sample separately, a CFA model was fit to both samples combined.

To determine if case-definition items exhibited DIF for SSc versus cancer, the Multiple- 
Indicator Multiple-Cause (MIMIC) model was utilized. The base MIMIC model consists of the CFA factor model, with the direct effect of group (SSc versus cancer) on the latent factor added. This serves to control for group differences on fatigue level. The MIMIC model also allows for adjustment for variables that may differ between comparison groups, by adding a direct effect on the latent factors. We controlled for differences in age.

Each item was regressed separately on the grouping variable to assess potential DIF. DIF is represented by a statistically significant $(\mathrm{P}<0.05)$ link of group with the item, controlling for differences in the overall level of the latent factor. If there was DIF for one or more items, the item with the largest magnitude of DIF was considered to have DIF, and the association between group and that item was included in the model. This procedure was repeated until no remaining items showed significant DIF. Once all items with significant DIF were identified, the magnitude of DIF items collectively was evaluated by comparing the difference on the latent factor between groups in the baseline model and after controlling for DIF. The magnitude of this difference was interpreted following Cohen's effect sizes, with $<=0.20 \mathrm{SD}$ indicating small, $0.50 \mathrm{SD}=$ moderate and 0.80 SD = large differences (39). Hommels' correction for multiple testing was applied (40).

A logistic regression procedure was utilized to assess whether Criterion B exhibited significant DIF (13). Thus, we assessed whether the Criterion B response (yes/no) was associated with group membership ( $\mathrm{SSc}$ versus cancer) after controlling for differences in the level of fatigue (symptoms A2-A10). In step 1, the total scale score for each patient was entered in the equation, following by the grouping variable to assess uniform DIF (step 2). Significant DIF was defined as the presence of a significant difference $(1-\mathrm{df}$ chi-square test; $\mathrm{P}<0.05)$ for the difference in chi-squared for the logistic regression between step 1 and step 2; and an effect size of at least 0.13 , defined as the difference in Nagelkerke's R-squared between steps 1 and 2 (13). 
An exploratory analysis was conducted to assess the impact of eliminating items with DIF on the prevalence of fatigue caseness in the SSc and cancer samples. The congruency of results using these alternative criteria and the original criteria was assessed.

CFA and MIMIC analyses were conducted using Mplus 7 (31) and all other analyses were conducted using IBM SPSS Statistics 20 (Chicago, IL).

\section{RESULTS}

\section{Sample characteristics}

Demographic and disease characteristics are displayed in Table 1.

SSc sample. In total, 345 SSc patients were contacted for an interview and 344 completed the Cancer-Related Fatigue interview, including 301 women. Of the 301 women, 10 were diagnosed with current MDD and not included in the present analysis. Thus, the SSc sample consisted of 291 female patients with a mean age of 58.1 years $(\mathrm{SD}=11.5)$ and mean time since diagnosis of 10.5 years $(\mathrm{SD}=8.5)$. Most patients $(\mathrm{n}=219,75.5 \%)$ were diagnosed with limited $(n=211,72.8 \%)$ or sine SSc $(n=8,2.8 \%)$. Seven patients $(2.4 \%)$ reported cancer comorbidity, and $45(15.4 \%)$ used immunosuppressant medication at the time of the interview. In total, 102 female patients (35.1\%) met criteria for Chronic Illness-Related Fatigue. The number of women who endorsed Criterion A and B items are displayed in Table 2.

Cancer sample. No women were excluded based on criterion C. Thus, the cancer sample consisted of 278 female patients without psychiatric disorder with a mean age of 57.9 years $(\mathrm{SD}=11.4)$ and a mean time post-treatment of 11.1 months $(\mathrm{SD}=6.6)$. Of these patients, 188 (67.6\%) had a negative lymph node status, and 90 (32.4\%) were positive. The majority $(\mathrm{n}=155$, $56.5 \%)$ underwent mastectomy, $123(43.5 \%)$ received conserving surgery, and $145(52.5 \%)$ 
received chemotherapy. Of the 278 women, 105 (37.8\%) met criteria for Cancer-Related Fatigue. The number of women who endorsed Criterion A and B items are displayed in Table 2.

\section{Confirmatory factor analysis}

A single-factor structure showed good fit in both samples $\left(\mathrm{SSc}: \mathrm{X}^{2}(27)=38.8, \mathrm{P}=0.07\right.$, $\mathrm{CFI}=0.94, \mathrm{TLI}=0.92, \mathrm{RMSEA}=0.06$; cancer: $\mathrm{X}^{2}(27)=28.1, \mathrm{P}=0.41, \mathrm{CFI}=1.00, \mathrm{TLI}=1.00$, RMSEA=0.02). Inspection of modification indices indicated that freeing error terms to covary would not improve model fit substantially.

\section{Differential Item Functioning}

Symptoms (A2-A10). The single-factor model was fit to the combined SSc and cancer samples, including a direct effect of group on the latent fatigue factor, as well as a direct effect of age on the latent fatigue factor, and fit well $\left(\mathrm{X}^{2}(43)=74.5, \mathrm{P}=0.002, \mathrm{CFI}=0.94\right.$, TLI=0.92, RMSEA=0.05). Prior to accounting for possible DIF, SSc patients had 0.05 SD higher latent factor scores (more fatigue) than cancer patients, although this difference was not statistically significant $(95 \%$ confidence interval $(\mathrm{CI})-0.23$ to $0.33, \mathrm{P}=0.71)$. As shown in Table 3 , there were two items with statistically significant DIF. Item 10 (short-term memory; $\mathrm{z}=3.18, \mathrm{P}=0.002$ ) and item 3 (trouble concentrating; $\mathrm{z}=3.45, \mathrm{P}<0.001$ ) were significantly less often endorsed by women with SSc compared to women with cancer with similar latent fatigue factor levels.

As shown in Table 3, after correcting for DIF for items 3 and 10, compared with the base model, there was an increase of $0.22 \mathrm{SD}$ on the latent fatigue factor in the difference between SSc and cancer. Thus, after correcting for DIF, women with SSc scored 0.27 SD higher on the latent fatigue factor $(95 \% \mathrm{CI}-0.03$ to $0.57, \mathrm{P}=0.08)$, a small to moderately higher level than women with breast cancer, although statistically non-significant. 
As a sensitivity analysis, we ran the MIMIC model with only the 7 items that had no statistically significant DIF, which yielded virtually the same results as the 9-item model corrected for the 2 DIF items, with a factor loading for group on the latent factor of 0.27 (95\% CI -0.03 to $0.56, \mathrm{P}=0.08)$.

Criterion $B$. In Step 1 of the logistic regression analysis (total score of symptoms A2 to A10), Nagelkerke's $\mathrm{R}^{2}$ was 0.217 . In Step 2, when the group variable was added to the model, Nagelkerke's $\mathrm{R}^{2}$ increased to $0.229\left(\Delta \mathrm{R}^{2}=0.012\right)$ which was not significant $\left(\mathrm{X}^{2}(1)=1.78, \mathrm{P}=0.18\right)$, suggesting that there was not DIF for Criterion B for SSc versus cancer patients.

\section{Case definition with and without DIF items}

As shown in Table 4, 97.5\% of the cancer sample was categorized consistently as a case or non-case when comparing the diagnosis based on the standard 5 of 9 symptom criterion and the revised 4 of 7 criterion, which excluded the 2 DIF items (Cohen's Kappa=0.95). The prevalence of cases of fatigue was similar in the SSc (35.1\% versus $36.1 \%)$ and cancer samples (37.9\% versus $37.4 \%$ ) using the two different criteria (Table 5).

\section{DISCUSSION}

The main finding was that items 3 (concentrating) and 10 (short-term memory) of the Cancer-Related Fatigue case-definition were significantly more frequently endorsed by breast cancer patients than women with SSc with similar levels of fatigue. If these two items, which appear to be metrically inequivalent across diseases, are omitted from the case-definition, and the case-definition is changed to Criterion A1 plus at least 4 out of the 7 remaining symptoms, the overall prevalence of Cancer-Related Fatigue in the cancer sample (37.4\%) was similar to the 
estimate when 5 of 9 symptoms were required (37.8\%). Furthermore, the status of individual patients changed for only 7 of 278 women $(2.5 \%)$.

Both items 3 and 10 are related to cognitive symptoms. A recent study reported that cognitive fatigue was present in $29 \%$ of cancer patients, and less common than physical fatigue $(57 \%)$ and emotional fatigue (37\%) (41). Cognitive fatigue could be related to cancer treatment, in particular chemotherapy, monoclonal antibody therapy and radiotherapy (42). A previous study in women successfully treated for breast cancer found that women meeting criteria for cancer-related fatigue performed worse on measures of verbal memory, sustained attention and reaction time compared to women not meeting criteria (31). Several centrally acting cytokines may contribute to this, specifically vascular endothelial growth factor and brain derived neurotrophic factor, both of which were elevated in the group with cancer-related fatigue compared to non-cases (31). These cytokines can disrupt the permeability of the blood brain barrier and provide a plausible biological mechanism for the central fatigue effects in cancer from an indirect tumor effect or secondary to treatment (43).

Authors of a recent systematic review of the Cancer-Related Fatigue criteria reported that it is unclear if the current requirement of a minimum of 5 out of 9 symptoms versus using a casedefinition based on fewer symptoms discriminates better between persons with and without cancer-related fatigue, and recommended investigating further refinement of the criteria (44). Based on our findings, items 3 and 10 do not add substantially to identifying cases among breast cancer patients. Because these items appear to be more related to cancer-related fatigue specifically, eliminating these items from the interview would facilitate the use of these criteria more broadly to assess Chronic Illness-Related Fatigue in other diseases, including rheumatic diseases. 
A unifying case definition of fatigue that can be applied broadly in medical populations would improve the comparability of results between diseases, which would facilitate research on etiology and interventions to reduce fatigue, as well as communication about fatigue among health professionals and patients. Recent Cochrane systematic reviews in cancer $(45,46)$ and rheumatoid arthritis (47), for instance, have identified that physical exercise and psychosocial interventions are promising approaches to addressing fatigue in these diseases. These types of programs can be quite resource and time-intensive, and should preferably be targeted to patients who may benefit most. Including patients with clinically significant fatigue, using the proposed case-definition, could increase the potential for improvement. Furthermore, coordinated efforts across investigations and diseases, including comparison and pooling of data, will strengthen the understanding and management of Chronic Illness-Related Fatigue.

As far as we know, no other studies have assessed the measurement equivalence of fatigue measures across medical diseases, which is surprising given that common measures have been routinely used across illnesses, including rheumatic diseases (48). Other measures of fatigue, in particular questionnaires that were initially designed for cancer-related fatigue and that include potentially cognitive fatigue items, may similarly have items that are less applicable and may distort results when being used more broadly in patients with other chronic diseases because similar scores obtained across these diseases may not reflect similar levels of fatigue.

There are limitations that should be considered in interpreting the results of this study. First, both samples constituted convenience samples, and only women were included in the present study. Thus, our sample may not reflect the full spectrum of the SSc and breast cancer populations. Second, the SSc and cancer samples were derived from two different countries, which may have led to difference in socio-cultural factors that could have influenced our results. 
Third, in the SSc sample, a phone interview was conducted to assess fatigue criteria, whereas a face-to-face interview was conducted in cancer. There are many examples of studies that have demonstrated the equivalency between structured diagnostic interviews conducted per telephone versus face-to-face interviews, for example in depression $(49,50)$, but this has not been established for the diagnostic fatigue interview. Fourth, although we excluded patients with depression, we did not exclude SSc patients with other psychiatric disorders that could have influenced fatigue, such as anxiety. Fifth, the case definition items were developed by experts, however, it is possible that there are other items that may better reflect fatigue in chronic illness. In addition, although there are clear commonalities between fatigue in cancer and other (rheumatic) conditions, there may also be components of fatigue that are disease-specific and that may not be captured by these generic fatigue criteria. As yet, very little is known about the fatigue experience of patients with SSc, however, that would guide refinement of criteria for SSc. Additional research to better understand fatigue experiences of patients with SSc, may facilitate work in this area. Finally, the assessment of DIF across the breast cancer and SSc samples is an important step in establishing the measurement properties of a generic Chronic Illness-related Fatigue case-definition. Future studies, however, should continue to examine other measurement properties, including aspects of reliability and validity, in scleroderma, across other rheumatic diseases and cancer, as well as examine DIF based on variables such as age, gender, and race/ethnicity.

In conclusion, the Cancer-Related Fatigue criteria can equivalently be used as a Chronic Illness-Related Fatigue case-definition in SSc if the cognitive fatigue symptoms are not included. If our results are replicated, in SSc and other populations, refining the Cancer-Related Fatigue criteria, particularly eliminating items 3 and 10, would facilitate research and the evaluation of 
interventions for fatigue in chronic diseases. Harmonized definitions of fatigue and the use of instruments with demonstrated measurement invariance across diseases will advance research and development and implementation of interventions in rheumatic diseases and other conditions. 


\section{References}

1. Nikolaus S, Bode C, Taal E, van de Laar MA. Fatigue and factors related to fatigue in rheumatoid arthritis: A systematic review. Arthritis Care Res 2013;65:1128-46.

2. Strickland G, Pauling J, Cavill C, McHugh N. Predictors of health-related quality of life and fatigue in systemic sclerosis: Evaluation of the EuroQol-5D and FACIT-F assessment tools. Clin Rheumatol 2012;31:1215-22.

3. Swain MG. Fatigue in chronic disease. Clin Sci 2000;99:1-8.

4. Fitzgibbon EJ, Murphy D, O'Shea K, Kelleher C. Chronic debilitating fatigue in Irish general practice: A survey of general practitioners' experience. Br J Gen Pract 1997;47:618-22.

5. Deale A, Wessely S. Patients' perceptions of medical care in chronic fatigue syndrome. Soc Sci Med 2001;52:1859-64.

6. Flowers N, Wolfe F. What do rheumatologists do in their practices? [abstract]. Arthritis Rheum 1998;41:337.

7. Vogelzang NJ, Breitbart W, Cella D, Curt GA, Groopman JE, Horning SJ, et al. Patient, caregiver, and oncologist perceptions of cancer-related fatigue: Results of a tripart assessment survey. The Fatigue Coalition. Semin Hematol 1997;34:4-12.

8. Passik SD, Kirsh KL, Donaghy K, Holtsclaw E, Theobald D, Cella D, et al. Patient-related barriers to fatigue communication: Initial validation of the fatigue management barriers questionnaire. J Pain Symptom Manage 2002;24:481-93.

9. Lynch J, Mead G, Greig C, Young A, Lewis S, Sharpe M. Fatigue after stroke: The development and evaluation of a case definition. J Psychosom Res 2007;63:539-44. 
10. Cella D, Davis K, Breitbart W, Curt G, Fatigue Coalition. Cancer-related fatigue: Prevalence of proposed diagnostic criteria in a United States sample of cancer survivors. J Clin Oncol 2001; $19: 3385-91$.

11. Cella D, Peterman A, Passik S, Jacobsen P, Breitbart W. Progress toward guidelines for the management of fatigue. Oncology 1998;12:369-77.

12. Teresi JA. Overview of quantitative measurement methods. Equivalence, invariance, and differential item functioning in health applications. Med Care 2006;44:S39-49.

13. Zumbo BD. A handbook on the theory and methods of differential item functioning (DIF):

Logistic regression modeling as a unitary framework for binary and likert-type (ordinal) item scores. Ottawa, ON: Directorate of Human Resources Research and Evaluation, Department of National Defense; 1999.

14. Wigley FM, Hummers LK. Clinical features of systemic sclerosis. In: Hochberg MC, Silman AJ, Smolen JS, Weinblatt ME, Weismann WH, eds. Rheumatology. Philadelphia: Mosby; 2003:1463-80.

15. Mayes MD, Lacey JV Jr, Beebe-Dimmer J, Gillespie BW, Cooper B, Laing TJ, et al. Prevalence, incidence, survival, and disease characteristics of systemic sclerosis in a large US population. Arthritis Rheum 2003;48:2246-55.

16. Suarez-Almazor ME, Kallen MA, Roundtree AK, Mayes M. Disease and symptom burden in systemic sclerosis: A patient perspective. J Rheumatol 2007;1718-26.

17. van Lankveld WG, Vonk MC, Teunissen H, van den Hoogen FH. Appearance self-esteem in systemic sclerosis-subjective experience of skin deformity and its relationship with physicianassessed skin involvement, disease status and psychological variables. Rheumatology 2007;46:872-6. 
18. Bassel M, Hudson M, Taillefer SS, Schieir O, Baron M, Thombs BD. Frequency and impact of symptoms experienced by patients with systemic sclerosis: Results from a Canadian national survey. Rheumatology 2011;50:762-7.

19. Hudson M, Steele R, Lu Y, Thombs BD, Canadian Scleroderma Research Group, Baron M. Work disability in systemic sclerosis. J Rheumatol 2009;36:2481-6.

20. Sandusky SB, McGuire L, Smith MT, Wigley FM, Haythornthwaite JA. Fatigue: An overlooked determinant of physical function in scleroderma. Rheumatology 2009;48:165-9. 21. Sandqvist G, Eklund M. Daily occupations-performance, satisfaction and time use, and relations with well-being in women with limited systemic sclerosis. Disabil Rehabil 2008;30:2735.

22. Alhajeri H, Hudson M, Fritzler M, Pope J, Tatibouet S, Markland J, et al. The 2013 ACR/EULAR classification criteria for systemic sclerosis outperform the 1980 criteria. Data from the Canadian scleroderma research group. Arthritis Care Res 2014; in press.

23. van den Hoogen F, Khanna D, Fransen J, Johnson SR, Baron M, Tyndall A, et al. 2013 classification criteria for systemic sclerosis: An American College of Rheumatology/European League Against Rheumatism collaborative initiative. Arthritis Rheum 2013;65:2737-47. 24. Minton O, Alexander S, Stone PC. Identification of factors associated with cancer related fatigue syndrome in disease-free breast cancer patients after completing primary treatment. Breast Cancer Res Treat 2012;136:513-20.

25. LeRoy EC, Black C, Fleischmajer R, Jablonska S, Krieg T, Medsger TA Jr, et al. Scleroderma (systemic sclerosis): Classification, subsets and pathogenesis. J Rheumatol $1988 ; 15: 202-5$. 
26. Kessler RC, Ustun TB. The World Mental Health (WMH) survey initiative version of the World Health Organization (WHO) Composite International Diagnostic Interview (CIDI). Int J Methods Psychiatr Res 2004;13:93-121.

27. American Psychiatric Association. Diagnostic and statistical manual of mental disorders, 4th ed, text revision. Washington, DC: American Psychiatric Association; 2000.

28. Alexander S, Minton O, Andrews P, Stone P. A comparison of the characteristics of diseasefree breast cancer survivors with or without cancer-related fatigue syndrome. Eur J Cancer $2009 ; 45 ; 384-92$.

29. First MB, Spitzer RL, Gibbon M, Williams JBW. Structured clinical interview for DSM-IVTR axis I disorders, research version, non-patient edition. New York: Biometrics Research, New York State Psychiatric Institute; 2001.

30. Minton O, Stone PC. A comparison of cognitive function, sleep and activity levels in diseasefree breast cancer patients with or without cancer-related fatigue syndrome. BMJ Support Palliat Care 2012;2:231-8.

31. Muthén LK, Muthén BO. Mplus user’s guide version 3.11. Los Angeles: Muthén LK \& Muthén BO;1998-2004.

32. Lai JS, Crane PK, Cella D. Factor analysis techniques for assessing sufficient unidimensionality of cancer related fatigue. Qual Life Res 2006;15:1179-90.

33. Tucker L, Lewis C. A reliability coefficient for maximum likelihood factor analysis. Psychometrika 1973;38:1-10.

34. Bentler PM. Comparative fit indexes in structural models. Psychol Bull 1990;107:238-46. 35. Steiger J. Structural model evaluation and modification: An interval estimation approach. Multivariate Behav Res 1990;25:173-80. 
36. Hu L, Bentler PM. Cutoff criteria for fit indexes in covariance structure analysis:

Conventional criteria versus new alternatives. Struct Equ Model 1999;6:1-55.

37. Kline RB. Principles and practice of structural equation modeling 2nd ed. New York:

Guilford Press; 2005.

38. Reise SP, Widaman KF, Pugh RH. Confirmatory factor analysis and item response theory:

Two approaches for exploring measurement invariance. Psychol Bull 1993;114:552-66.

39. Cohen J. Statistical power analysis for the behavioral sciences. Hillsdale: Lawrence Erlbaum Associates; 1988.

40. Hommel G. A stagewise rejective multiple test procedure on a modified Bonferroni test. Biometrika 1988;75:383-6.

41. James S, Wright P, Scarlett C, Young T, Jamal H, Verma R. Cancer-related fatigue: results from patient experience surveys undertaken in a UK regional cancer centre. Support Care Cancer. [Epub ahead of print]

42. Correa DD, Ahles TA. Neurocognitive changes in cancer survivors. Cancer J 2008;14:396400.

43. Minton O, Coulton GR, Stone P. Multi-analyte profiling and pathway analysis of plasma for proteins associated with cancer-related fatigue syndrome in disease-free breast cancer patients after primary treatment. BMJ Support Palliat Care 2014;4:349-56.

44. Donovan KA, McGinty HL, Jacobsen PB. A systematic review of research using the diagnostic criteria for cancer-related fatigue. Psychooncology 2013;22:737-44.

45. Cramp F, Byron-Daniel J. Exercise for the management of cancer-related fatigue in adults. Cochrane Database Syst Rev 2012;11:CD006145.

46. Goedendorp MM, Gielissen MF, Verhagen CA, Bleijenberg G. Psychosocial interventions 
for reducing fatigue during cancer treatment in adults. Cochrane Database Syst Rev 2009;1:CD006953.

47. Cramp F, Hewlett S, Almeida C, Kirwan JR, Choy EH, Chalder T, et al. Nonpharmacological interventions for fatigue in rheumatoid arthritis. Cochrane Database Syst Rev 2013;8:CD008322.

48. Hewlett S, Dures E, Almeida C. Measures of fatigue: Bristol Rheumatoid Arthritis Fatigue Multi-Dimensional Questionnaire (BRAF MDQ), Bristol Rheumatoid Arthritis Fatigue Numerical Rating Scales (BRAF NRS) for severity, effect, and coping, Chalder Fatigue Questionnaire (CFQ), Checklist Individual Strength (CIS20R and CIS8R), Fatigue Severity Scale (FSS), Functional Assessment of Chronic Illness Therapy (Fatigue) (FACIT-F), Multidimensional Assessment of Fatigue (MAF), Multi-dimensional Fatigue Inventory (MFI), Pediatric Quality of Life (PedsQL) multi-dimensional fatigue scale, Profile of Fatigue (ProF), Short Form-36 Vitality subscale (SF-36 VT), and Visual Analog Scales (VAS). Arthritis Care Res 2011;63:S263-86.

49. Rohde P, Lewinsohn PM, Seeley JR. Comparability of telephone and face-to-face interviews in assessing axis I and II disorders. Am J Psychiatry 1997;154:1593-8.

50. Muskens EM, Lucassen P, Groenleer W, van Weel C, Oude Voshaar R, Speckens A. Psychiatric diagnosis by telephone: is it an opportunity? Soc Psychiatry Psychiatr Epidemiol 2014;49:1677-89. 